\title{
Amiodarone Prevents Atrial Fibrillation Induced by Arsenic Trioxide in Extramedullary Plasmacytoma Involving Lumbar Disc: A Novel Case Report
}

\section{Shakil Md A* and Liu S}

Department of Hematology, Zhongnan Hospital of Wuhan University, China

${ }^{*}$ Corresponding author: Shakil Md A, Department of Hematology, Zhongnan Hospital of Wuhan University, China, E-mail: ahanafshakil@yahoo.com

Citation: Shakil Md A, Liu S (2020) Amiodarone Prevents Atrial Fibrillation Induced by Arsenic Trioxide in Extramedullary Plasmacytoma Involving Lumbar Disc: A Novel Case Report. J Hematol Blood Disord 6(1): 102. doi: 10.15744/2455-7641.6.102

\section{Received Date: January 18, 2020 Accepted Date: February 3, 2020 Published Date: February 5, 2020}

\begin{abstract}
Extramedullary plasmacytoma (EMP), characterized by infiltration of malignant plasmablastic clones outside the bone marrow in absence of multiple myeloma. Accounting for approximately $4 \%$ of all plasma cell dyscrasias, it has a predilection for head and neck region. However, in our presenting case we describe a 59-year-old man with primary plasmacytoma involving lumbar disc which is an exceedingly rare phenomenon. Antineoplastic drug arsenic trioxide (ATO) is predominantly a chemotherapeutic agent for acute promyelocytic leukemia has shown an immense cytotoxic effect on plasmacytoma. There's a growing recognition that enhanced therapeutic efficacy made ATO propitious for treating progressive and refractory EMP. After reviewed the existing literature, it's noteworthy that ATO acts as a trigger for cardiovascular diseases such as cardiac fibrosis, long QT syndrome, cardiotoxicity, torsades de pointes, ventricular tachycardia, cardiac fibroblast apoptosis, complete atrioventricular block, and sudden death. Inversely, based on potency and various practice guidelines, amiodarone is recommended for treating arrhythmias such as conversion of atrial fibrillation (AFib) to sinus rhythm or ventricular tachycardia. Herein we present a case of ATO induced AFib in a primary lumbar disc plasmacytoma diagnosed patient and amiodarone to prevent it.
\end{abstract}

Keywords: Extramedullary Plasmacytoma (EMP); Arsenic Trioxide (ATO); Amiodarone; Atrial Fibrillation (AFib); Lumbar disc plasmacytoma

\section{Introduction}

Plasma cell dyscrasia is an assortment of hematological malignancies arising from excessive monoclonal proliferation of neoplastic plasma cells and detection of a monoclonal immunoglobulin protein (M component). These malignancies include: multiple myeloma (MM) (most common), solitary osseous plasmacytoma, plasma cell leukemia, Waldenström macroglobulinemia, amyloidosis, and extramedullary plasmacytoma. Inversely, precursors of MM (i.e., monoclonal gammopathy of undetermined significance (MGUS) and smoldering multiple myeloma) are benign. There is a growing realization that the mechanism of the MM involves stochastic progression and complex changes in both the bone marrow malignant plasmacytes and in focal bone association, principally vertebrae, ribs, sternum, pelvis or skull. However, extramedullary plasmacytoma (EMP) is defined by infiltration of malignant plasmablastic clones outside the bone marrow or adjacent soft tissue, without evidence of MM. The most overt manifestation site of EMPs is upper respiratory tract region (83.2\%) (i.e., pharynx (22.1\%), paranasal sinuses (12.4\%), nasal cavity (18.7\%), oral cavity $(15.3 \%)$, and eye/brain $(3.2 \%)$ and other regions such as gastrointestinal tract (8.9\%), skin (6.6\%), lymph nodes (4.8\%), pleura (1.3\%), testes $(1.6 \%)$, endocrine glands $(1.4 \%)$, liver (1.1\%), and peritonium (1.5\%). For EMPs, incidence rates (IRs) were highest in Blacks (0.18\%), intermediate in Whites (0.11\%), and lowest in Asian/Pacific Islanders (0.07\%). Very rarely, plasmacytoma can occur in lumbar intervertebral disc, causing tremendous lower extremity weakness. In an investigation of 871 cases, devised by $\mathrm{C}$. Alexiou et al., approximately 15.1\% of EMP patients eventually develop MM [1]. A high incidence of extramedullary relapses was observed in almost all patients who underwent autologous stem cell transplantation (ASCT). By contrast, new treatment strategies including immunomodulatory drugs (IMiDs: thalidomide, lenalidomide, pomalidomide), proteosome inhibitors (PIs: ixazomib, carfilzomib, oprozomib, and marizomib), and other molecularly-targeted therapies directed at specific cell-signaling pathways (including: histone deacetylase inhibitors, cell-cycle inhibitors, kinesin spindle-protein inhibitors) are demonstrating high efficacy in terms of cancer control in refractory MM patients [2]. Most recently U.S. Food and Drug Administration (FDA) approved Janssen and Genmab's Darzalex (daratumumab) in combination with lenalidomide and dexamethasone as a first-line treatment for MM patients who are incapable of ASCT. 
Arsenic trioxide (ATO) is used as an active single agent to inhibit growth and achieve complete remissions in both newly diagnosed and relapsed acute promyelocytic leukemia (APL) patients. On a detailed literature review, it can also induce apoptosis in chronic myelogenous leukemia cells, some solid cancer tumor cells such as esophageal cancer, breast cancer, and neuroblastoma, malignant lymphocytic cell lines, and myeloma cells [3]. Enhanced therapeutic efficacy made ATO propitious for treating progressive and refractory EMP. Numerous mechanisms are described for the therapeutic effect of novel agent ATO: antiproliferation of myeloma cells by inducing cell cycle arrest and activation of pro-caspase-3 [4,5]; inhibition of angiogenesis via targeting forkhead box 03 (FOXO3) gene in gastric cancer [6]; induce programmed cell death in APL by degradation of promyelocytic leukemia (PML) protein and PML-retinal acid receptor alpha (PML-RARa) fusion proteins [7]; growth inhibition in myeloid leukemia by cross linking tubulin and inactivating GTP binding site [8]; modulation of glutathione redox system promotes apoptosis in B-cell lymphoma [9]. Nevertheless, we report a case of arsenic trioxide induced atrial fibrillation and amiodarone as an ideal drug to prevent it.

\section{Case presentation}

A 59-year-old Chinese man with numbness and paresthesia (burning, tingling sensation) in left lower extremity for one month presented to the hospital. The patient reported experiencing these altered sensations around 2 or 3 pm in a paroxysmal manner, and transiently relieved after nighttime rest. Tracing back his physical history, he complained of a mild intermittent back pain and right knee swelling. Medical evaluation at presentation revealed normal muscle strength and tension, normal cognitive function, no yellow pigmentation of skin and sclera, superficial lymph nodes were normal, and normal thyroid gland. Bilateral lungs auscultation were clear; no cracles, stridor, rhonchi, or pleural rub. Cardiovascular exam was within ordinary limits with no murmurs on auscultation, no distention of jugular vein was observed even peripheral edema was not perceivable. Abdominal and renal physical examinations both were normal.

After admission, magnetic resonance imaging (MRI) showed a well defined $5^{\text {th }}$ lumbar disc tumor resulting cauda equina nerve compression. An extramedullary plasmacytoma (EMP) was diagnosed on intervertebral tumor biopsy with infiltration of at least $40 \%$ of plasma cells (Figure 1A and B). His immunohistochemistry profile of lumbar disc plasmacytoma was indicative of EMP (positive for CD38, CD138, MUM-1, BOB.1, CD79A, BCL-2, OCT2, C-MYC, P53, $\kappa>>\lambda$; negative for CD3, CD43, CD20, PAX5, CD5, CD10, BCL-6, CyclinD1, CD21, CD23, a-Syn, SOX11). Simultaneously, the bone marrow biopsy that manifested clonal $5 \%$ of plasma cells was distinctive (positive for HLA-DR ${ }^{\mathrm{dim}}, \mathrm{CD} 34, \mathrm{CD} 13, \mathrm{CD} 33^{\mathrm{dim}}$; negative for CD15, CD64, CD14, CD16). Fluorescence in situ hybridization (FISH) from bone marrow denoted normal karyotype (46, XY) (Table 1).

\begin{tabular}{|c|c|c|}
\hline \multicolumn{3}{|c|}{ Hematological values } \\
\hline Parameters & Patient's values & Reference values \\
\hline Red blood cells & $2.56 \times 10^{12} / \mathrm{L}$ & $4.30-5.80 \times 10^{12} / \mathrm{L}$ \\
\hline White blood cells & $2.93 \times 10^{9} / \mathrm{L}$ & $3.50-9.50 \times 10^{9} / \mathrm{L}$ \\
\hline Platelets & $150 \times 10^{9} / \mathrm{L}$ & $125-350 \times 10^{9} / \mathrm{L}$ \\
\hline Hemoglobin & $66 \mathrm{~g} / \mathrm{L}$ & $130-175 \mathrm{~g} / \mathrm{L}$ \\
\hline Neutrophils & $1.46 \times 10^{9} / \mathrm{L}$ & $1.80-6.30 \times 10^{9} / \mathrm{L}$ \\
\hline Monocytes & $10.2 \%$ & $3 \%-10 \%$ \\
\hline Lymphocytes & $1.03 \times 10^{9} / \mathrm{L}$ & $1.10-3.20 \times 10^{9} / \mathrm{L}$ \\
\hline Mean corpuscular volume (MCV) & $85.2 \mathrm{fL}$ & $82-100 \mathrm{fL}$ \\
\hline C-reactive protein (CRP) & $4.1 \mathrm{mg} / \mathrm{L}$ & $0-10 \mathrm{mg} / \mathrm{L}$ \\
\hline Erythrocyte Sedimentation Rate (ESR) & $103 \mathrm{~mm} / \mathrm{H}$ & $0-15 \mathrm{~mm} / \mathrm{H}$ \\
\hline Calcium & $2.51 \mathrm{mmol} / \mathrm{L}$ & $2.15-2.57 \mathrm{mmol} / \mathrm{L}$ \\
\hline Prothrombin time & $15.4 \mathrm{~s}$ & $11.5-14.5 \mathrm{~s}$ \\
\hline Prothrombin activity & $73 \%$ & $75 \%-125 \%$ \\
\hline International normalized ratio (INR) & 1.22 & $0.80-1.20$ \\
\hline \multicolumn{3}{|c|}{ Urinalysis } \\
\hline $\begin{array}{l}\text { Estimated Glomerular Filtration Rate } \\
\text { (eGFR) }\end{array}$ & $\begin{array}{c}100.9 \mathrm{ml} / \\
\mathrm{min} / 1.73 \mathrm{~m}^{2}\end{array}$ & $>90 \mathrm{ml} / \mathrm{min} / 1.73 \mathrm{~m}^{2}$ \\
\hline Creatinine & $69 \mu \mathrm{mol} / \mathrm{L}$ & $59-104 \mu \mathrm{mol} / \mathrm{L}$ \\
\hline Urea & $5.3 \mathrm{mmol} / \mathrm{L}$ & $1.7-8.3 \mathrm{mmol} / \mathrm{L}$ \\
\hline Uric acid & $384 \mu \mathrm{mol} / \mathrm{L}$ & $202.3-416.5 \mu \mathrm{mol} / \mathrm{L}$ \\
\hline
\end{tabular}




\begin{tabular}{|c|c|c|}
\hline \multicolumn{3}{|c|}{ Hepatic function tests } \\
\hline Total protein & $91.4 \mathrm{~g} / \mathrm{L}$ & $64-83 \mathrm{~g} / \mathrm{L}$ \\
\hline Alkaline phosphatase (ALP) & $38 \mathrm{U} / \mathrm{L}$ & $40-130 \mathrm{U} / \mathrm{L}$ \\
\hline Glucose & $5.01 \mathrm{mmol} / \mathrm{L}$ & $4.11-6.05 \mathrm{mmol} / \mathrm{L}$ \\
\hline Albumin & $32.5 \mathrm{~g} / \mathrm{L}$ & $35-52 \mathrm{~g} / \mathrm{L}$ \\
\hline \multicolumn{2}{|c|}{ Serum protein electrophoresis } \\
\hline Parameters & Patient's values & Reference values \\
\hline IgG & $43.5 \mathrm{~g} / \mathrm{L}$ & $7.51-15.6 \mathrm{~g} / \mathrm{L}$ \\
\hline IgA & $0.21 \mathrm{~g} / \mathrm{L}$ & $0.82-4.53 \mathrm{~g} / \mathrm{L}$ \\
\hline IgM & $0.27 \mathrm{~g} / \mathrm{L}$ & $0.46-3.04 \mathrm{~g} / \mathrm{L}$ \\
\hline$\kappa$ free light chain (FLC) concentration & $283.7 \mathrm{mg} / \mathrm{L}$ & $3.30-19.40 \mathrm{mg} / \mathrm{L}$ \\
\hline$\lambda$ FLC concentration & $8.6 \mathrm{mg} / \mathrm{L}$ & $5.71-26.30 \mathrm{mg} / \mathrm{L}$ \\
\hline$\kappa / \lambda$ ratio & 32.988 & $0.26-1.65$ \\
\hline Albumin & $44.7 \%$ & $59.8 \%-72.4 \%$ \\
\hline$\beta_{2}$ Microglobulin & $3.36 \mathrm{mg} / \mathrm{L}$ & $0.8-2.4 \mathrm{mg} / \mathrm{L}$ \\
\hline$\gamma$-globulin & $0.7 \%$ & $8 \%-15.8 \%$ \\
\hline \multicolumn{2}{|c|}{ Urine protein electrophoresis } \\
\hline Total $\kappa$ light chain & $1010 \mathrm{mg} / \mathrm{L}$ & $0-6.88 \mathrm{mg} / \mathrm{L}$ \\
\hline Total $\lambda$ light chain & $18.50 \mathrm{mg} / \mathrm{L}$ & $0-3.78 \mathrm{mg} / \mathrm{L}$ \\
\hline$\kappa / \lambda$ ratio & 54.595 & $0.75-4.50$ \\
\hline
\end{tabular}

Table 1: Hematological values, hepatic function tests, urinalysis, serum and urine electrophoresis results

The patient was subsequently subjected to skeletal scintigraphy with SPECT (single photon emission computed tomography).

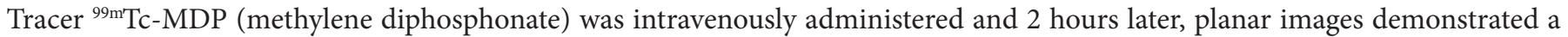
number of hot spots in bilateral shoulder joints, bilateral clavicles, bilateral costal cartilages, bilateral hip joints, and bilateral ischia. Thus, EMP was confirmed as IgG- $\kappa$, Durie Salmon IIIA, International Staging System I.

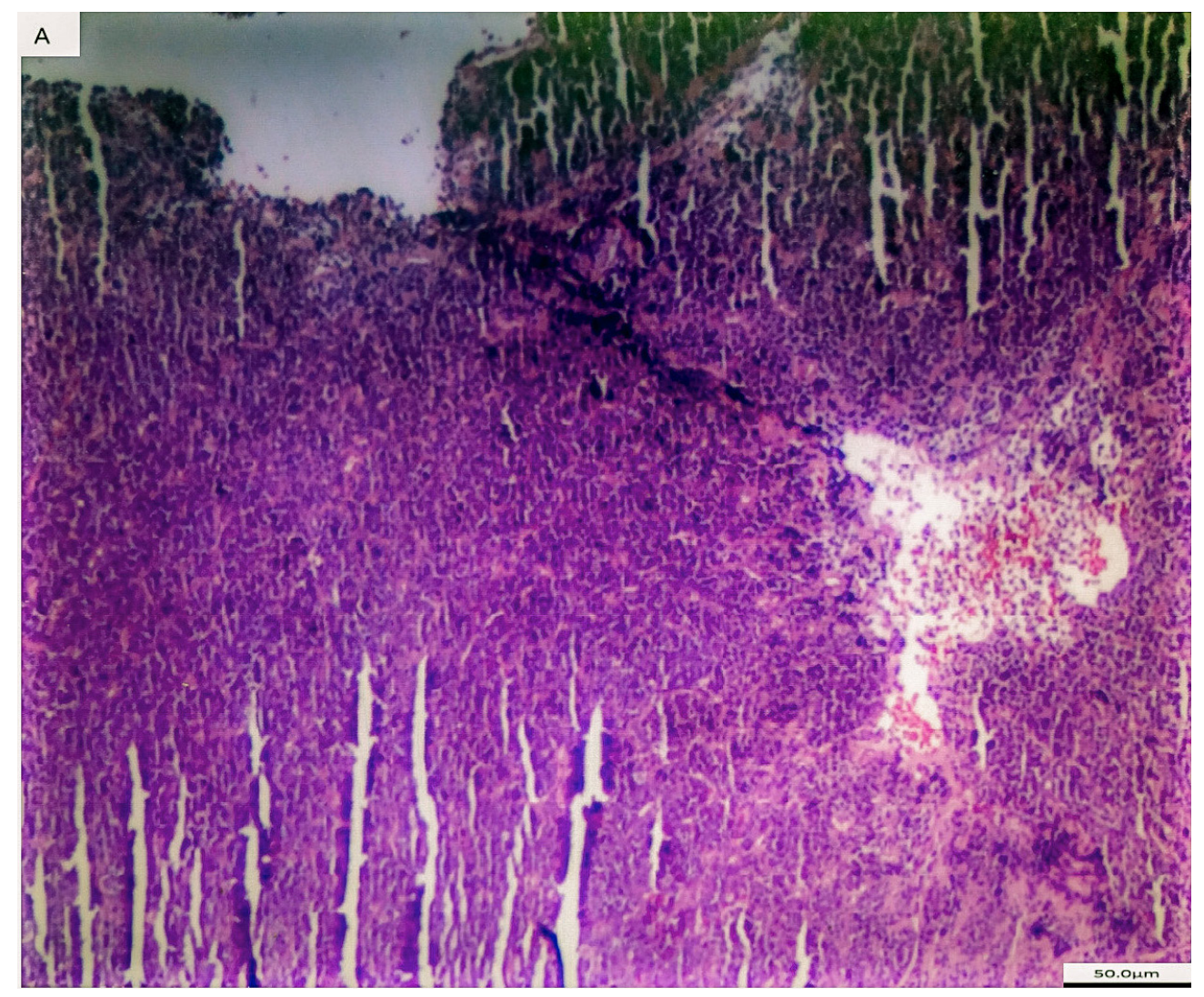




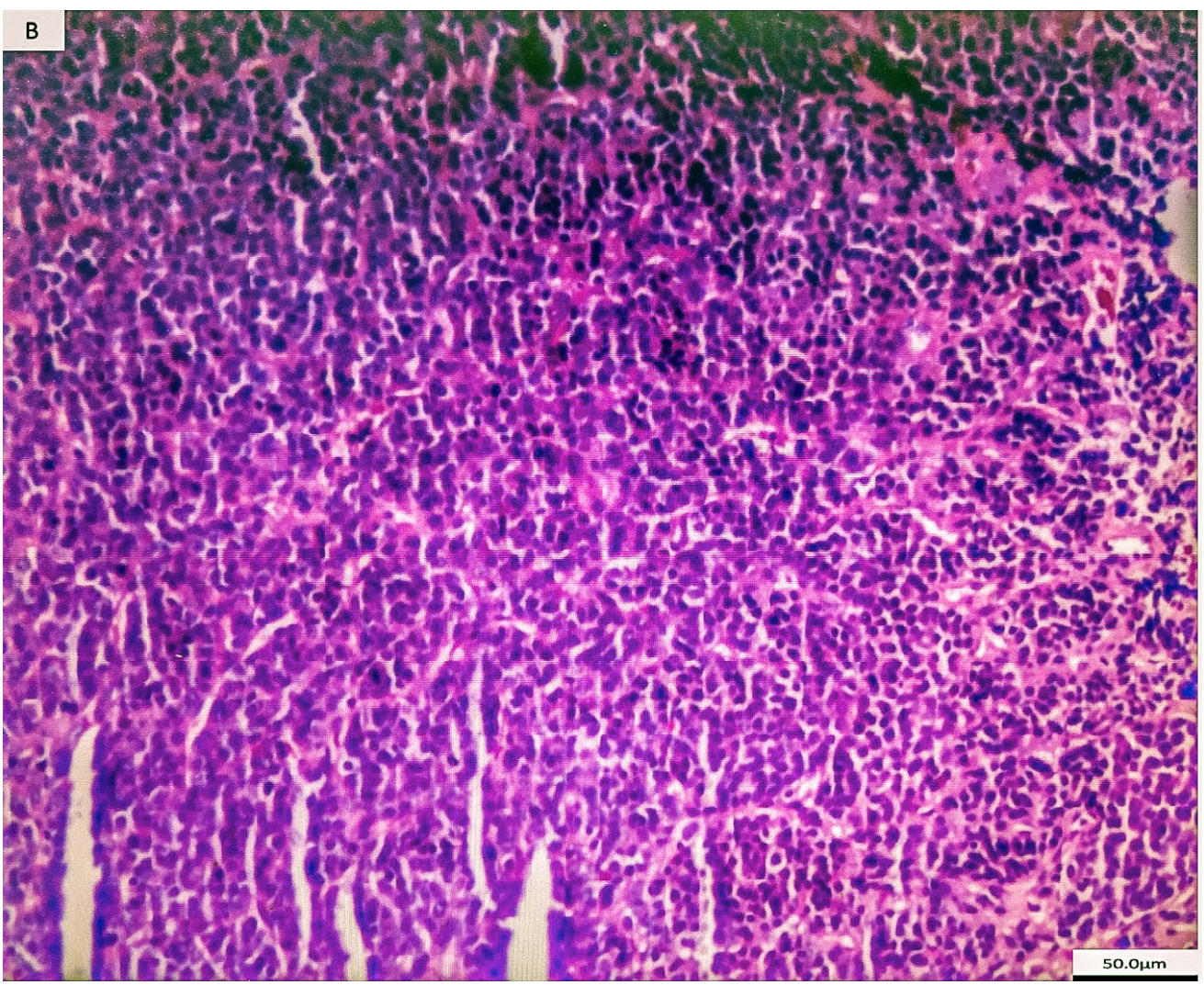

Figure 1: $(\mathbf{A}$ and $\mathbf{B})$ The $5^{\text {th }}$ lumbar disc plasmacytoma was approximately $2 \mathrm{~cm} \times 2 \mathrm{~cm} \times 0.5 \mathrm{~cm}$. Its high power scanner view (H\&E $\times 400$ magnification) shows proliferation of immature plasma cells

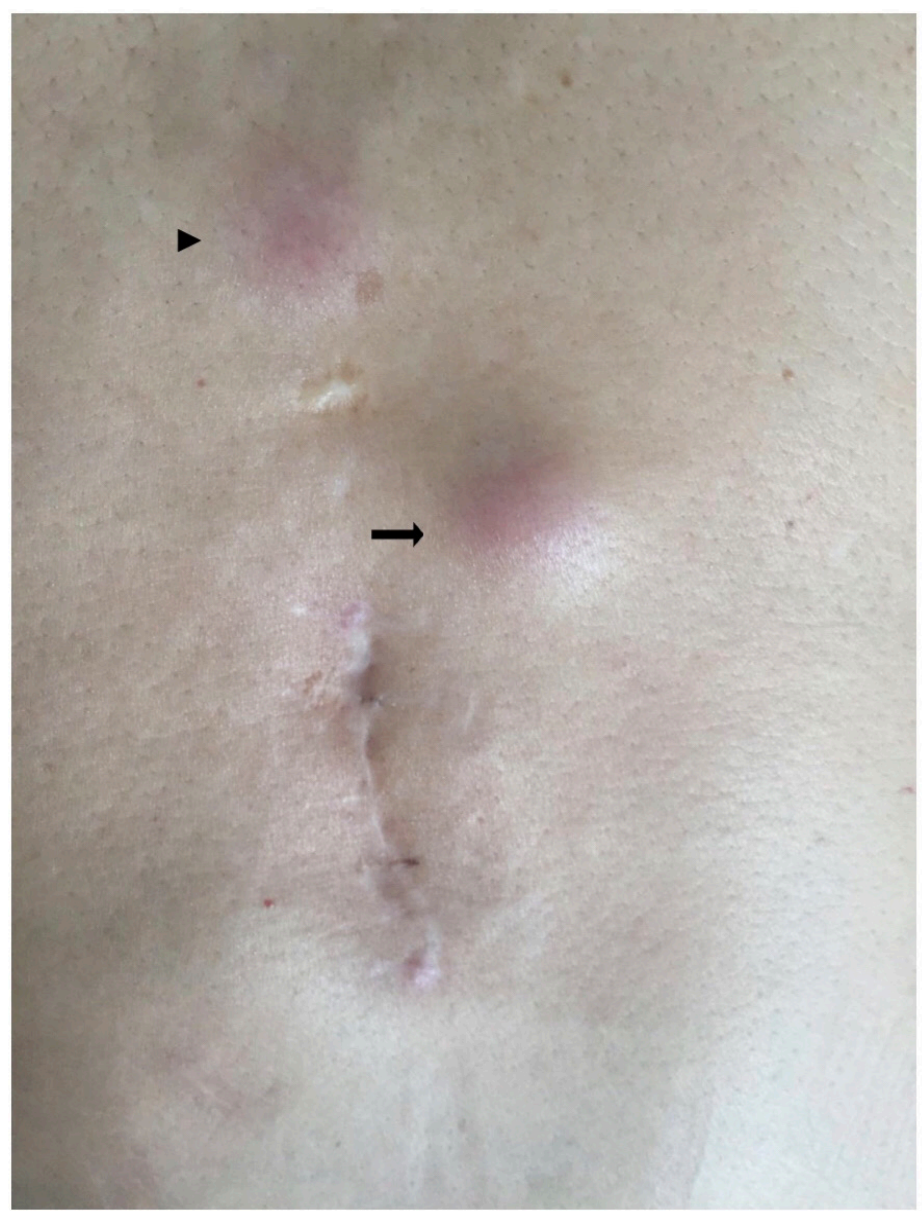

Figure 2: Recurrent extramedullary plasmacytoma on lower back (arrow: $1.8 \mathrm{~cm}$ and arrowhead: $1.1 \mathrm{~cm}$ in diameters). The scar is due to the lumbar disc plasmacytoma surgery 


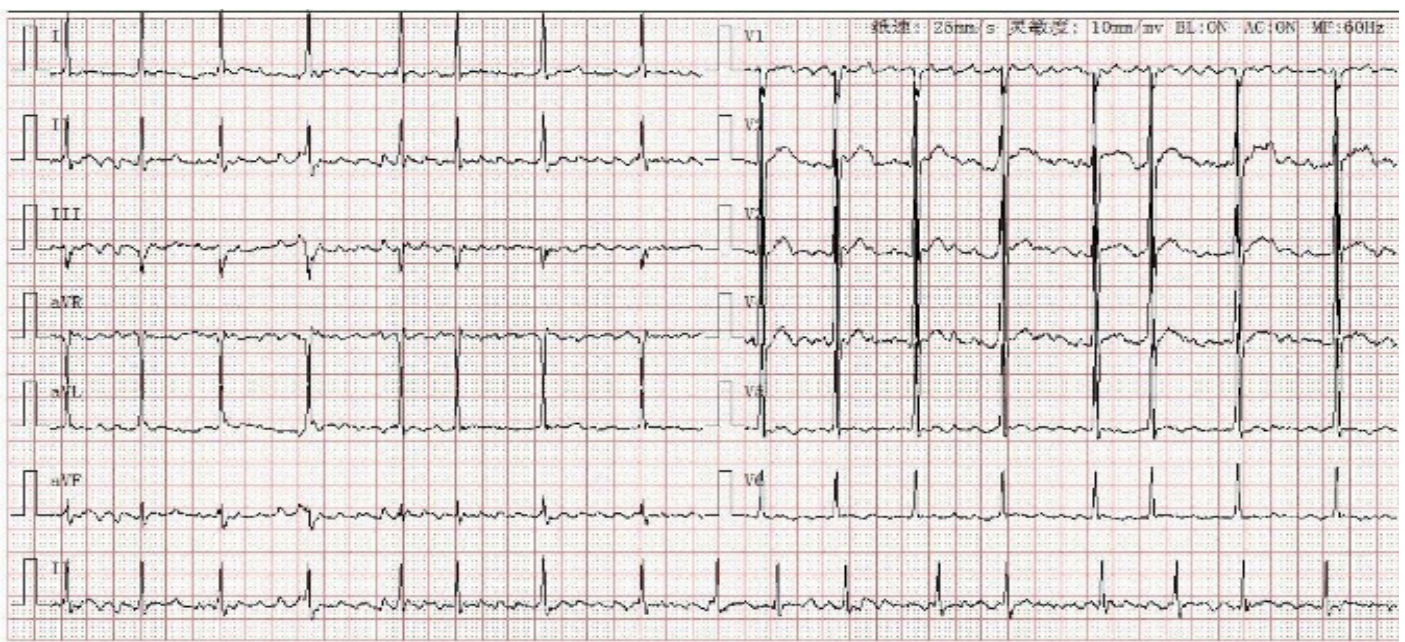

Figure 3: One of the atrial fibrillation relapses after induction therapy

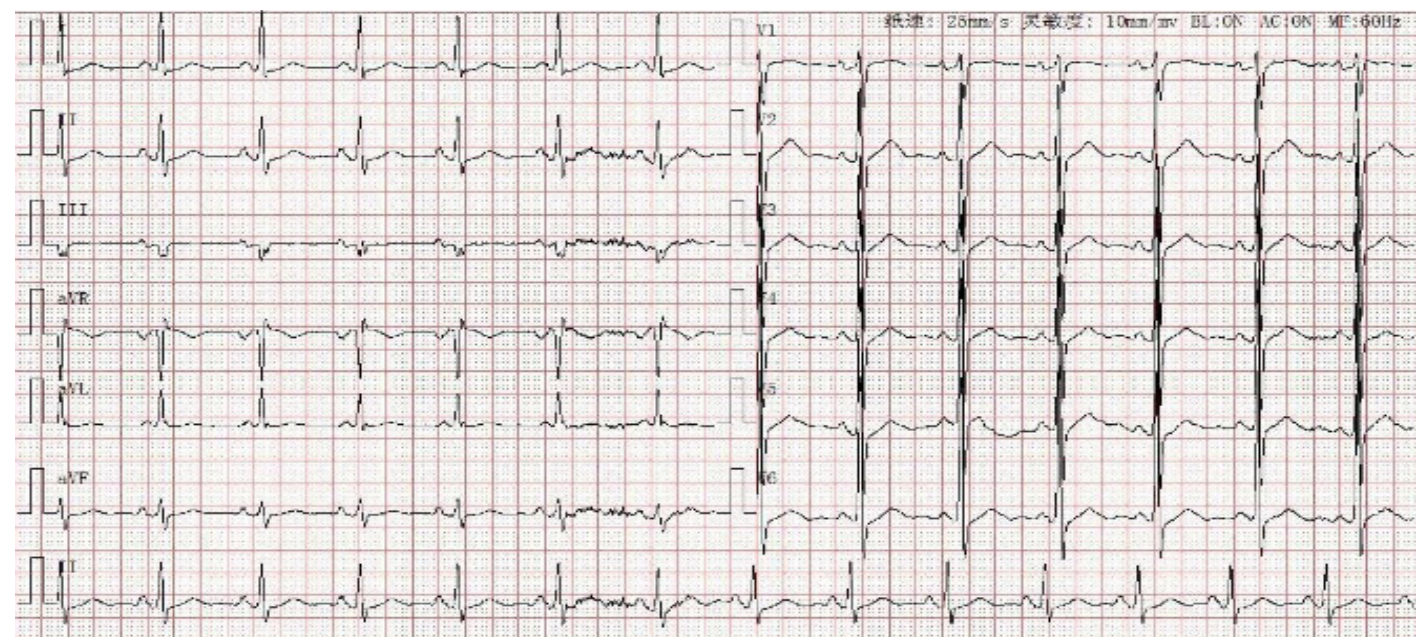

Figure 4: Amiodarone prevents arsenic trioxide induced atrial fibrillation

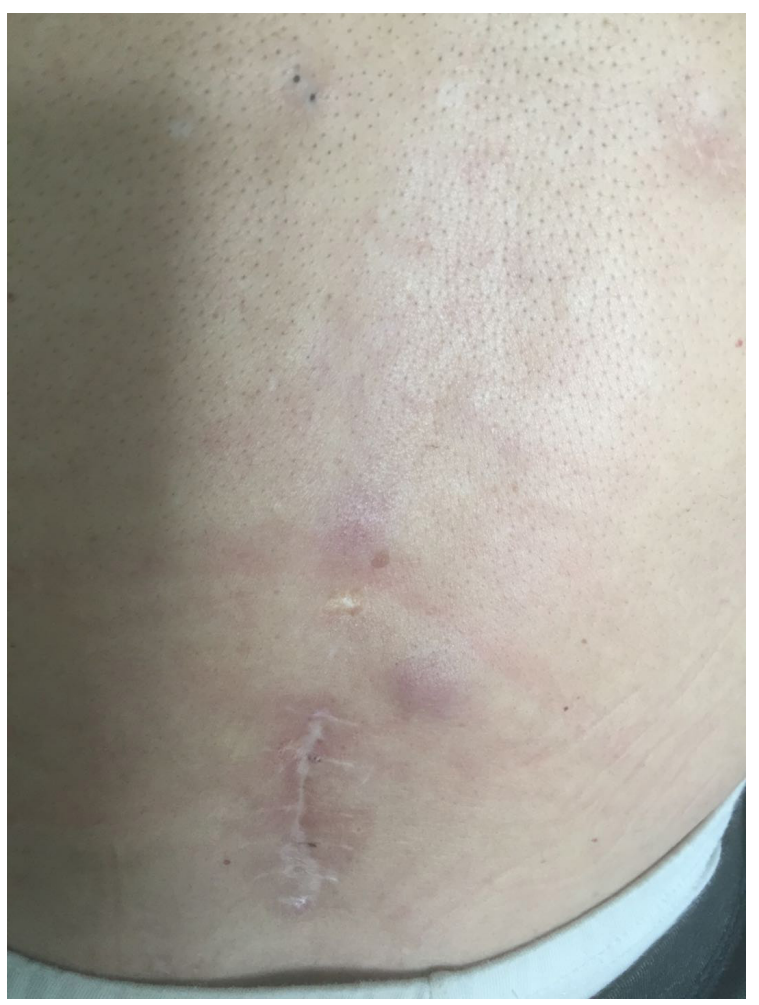

Figure 5: Arsenic trioxide as an effective chemotherapeutic agent in extramedullary plasmacytoma; Patient's nodule was disappeared 
The patient was scheduled to get five-cycle chemotherapy (bortezomib, dexamethasone). First four cycles were much effective with less recovery periods; however, during the fifth cycle patient developed myelosuppression, numbness in upper and lower extremities, and respiratory infection. After the induction therapy he was started to treat with LCD regimen (lenalidomide 20mg on days 1-21; cyclophosphamide $600 \mathrm{mg}$ on days $1,8,15$; dexamethasone $20 \mathrm{mg}$ on days 1-4, days 9-12, days 17-20) along with arsenic trioxide (ATO) $10 \mathrm{mg}$ on days 1-28. While there was a major improvement in disease condition, the recurrent plasmacytoma (shown as swelling or nodule) originated from subcutaneous tissue on his lower back. Red, soft consistency, smooth margin, non-tender and round are among the examined features of this nodule (Figure 2). The third cycle of therapy he developed symptoms of heart palpitations, dyspnea, polydipsia, and dizziness. Electrocardiogram (EKG) revealed evidence of atrial fibrillation (AFib) (Figure 3). Suspecting further deterioration of cardiac conditions, we discontinued chemotherapy; moreover, electrical cardioversion was used to reset cardiac arrhythmia to sinus rhythm. The fourth cycle of therapy, ATO was reintroduced with a reduced dose of $5 \mathrm{mg}$; additionally, certain medications were given him to protect stomach, nourish nerves, balance electrolytes, and promote microcirculation. On Day 7 of the therapy he complained of discomfort in lower xiphoid process. An immediate EKG pointed out atrial flutter accompanied with fast ventricular rate and on the same day chemotherapy was discontinued. This time we again performed cardioversion procedures, furthermore, administered intravenous amiodarone. The fifth cycle of therapy patient was disoriented, panicked, and agitated which led an EKG of AFib relapse and ST-T pathologically changed leads. As a result, chemotherapy was canceled immediately and on the same day he was administrated intravenous anticoagulants as well was given amiodarone $200 \mathrm{mg}$ twice a day. Despite the patient during cardioversion developed embolism risk factors, he left hospital later. It was almost one year since his extramedullary plasmacytoma was diagnosed, the sixth cycle of therapy we included pomalidomide. But he had AFib relapse throughout the chemotherapy sessions. A quick electrical cardioversion restored the regular rhythm and he was suggested oral amiodarone $200 \mathrm{mg}$ three times a day. Lastly, the seventh cycle of an antiarrhythmic-antineoplastic combined therapy (LCD, ATO and amiodarone) was successful without AFib recurrence (Figure 4). In addition, his back's nodule was disappeared (Figure 5) and no tenderness was recorded.

\section{Discussion}

Extramedullary plasmacytoma (EMP), clinical aspects are exceedingly heterogenous, and their management is certainly challenging. Conferring adverse prognosis, new tools (e.g., cytogenetics, PET-CT, immunophenotyping, electrophoretic assay, MRI) for diagnosis and monitoring of EMP are advancing much faster. Histopathologically, biopsies are most important when making a definite diagnosis.

It's noteworthy that there are some compelling findings in our case. Firstly, extramedullary plasmacytoma (EMP) involving lumbar intervertebral disc; secondly arsenic trioxide (ATO) is an essential treatment for EMP but can induce atrial fibrillation (AFib); thirdly increased dosage of amiodarone prevents AFib induced by ATO.

Extramedullary plasmacytoma accounts for 1\%-4\% of all plasma cell neoplasia and has a predilection for the head and neck region such as forehead, temporal lobe, nasopharynx, paranasal sinuses, tonsils, and laryngeal cleft. Seldom but clinically serious other anatomical locations are trachea, lung, lymph nodes, spleen, breast, gastrointestinal tract urogenital tract and so on. Remarkably, another type of primary plasmacytoma named solitary osseous plasmacytoma (SOP) is a biopsy proven osteolytic bone tumors in absence of MM and EMP. Marrow containing bones especially spine is typical site for SOP. Some recent published cases of spinal plasmacytoma like thoracic (T7-T9), cervical (C3), lumbar (L4) confirm this anatomical region and involvement with malignancy [10-12]. In our paper, we described an exceptional case of primary plasmacytoma with lumbar disc involvement. According to its atypical phenomenon, EMP can be easily misdiagnosed in outpatient care. In such manner, hospital admission is important for evaluate the potential diseases and establish essential diagnostic tests (e.g., complete blood count/metabolic panel, serum/urine electrophoresis, skeletal survey, fluorescences in situ hybridization). Many spinal and systemic conditions may mimic lumbar disc plasmacytoma's symptoms. For example, lumbar strain, herniated spinal disc, spinal stenosis, MM, cauda equina syndrome, pyelonephritis, lymphoma, multiple sclerosis, leukemia, or Gitelman's syndrome.

Plasmacytoma is commonly associated with anemia (low red blood cells), leukopenia (low white blood cells), and thrombocytopenia (low platelets). Overproduction of plasma cells, interleukins (IL-1 and IL-6), and tumor necrosis factor alpha (TNF- $\alpha$ ) are capable of suppressing erythropoietin (EPO) production. In our patient, the implications of anemia was obvious such as reduced hemoglobin, hematocrit, dyspnea, and ischemic complications. However, his thrombocytes was within normal range which showed negative signs of common bleeding disorders (e.g., acquired hemophilia, immune-thrombocytopenic purpura (ITP), amegakaryocytic thrombocytopenia, hemolytic uremic syndrome (HUS), and myelodysplastic syndrome). Hypercalcemia in plasma cell neoplasms is another frequent metabolic complication, ultimately caused by bone destruction from local osteolytic tumor lesions. Our patient's skeletal, digestive, and endocrine (i.d.,parathyroid, thyroid and adrenal glands) systems are intact, routine follow up blood tests and radiographs revealed no osteolysis activity. In this describing case, patient has hyperviscosity due to abnormal production of IgG and light chain kappa, $\kappa(\mathrm{IgG}=43.5 \mathrm{~g} / \mathrm{L}, \kappa=283.7 \mathrm{mg} / \mathrm{L})$ causes somnolence, polydipsia, blurred vision, listless-ness, headache, and agitation. His hepatic function tests (i.d., albumin, ALP (alkaline phosphatase), ALT (alanine aminotransferase), AST (aspartate aminotransferase), gamma-glutamyl transpeptidase (GGT), prothrombin time (PT/INR), bilirubin) and urinalysis tests (i.d., eGFR, creatinine, urea, uric acid) are normal. 
Arsenic, designated as "king of poisons" due to its toxicity in human beings. The health of billions of people has been threatened by arsenic exposure from ingestion of contaminated water or food, inhalation of industrial air or sawdust, and use of agricultural herbicides. Chronic arsenic exposure has long been recognized carcinogenic to humans that can induce malignancies of skin, lung, liver, kidney, urinary bladder, bone, larynx, and prostate. Even though arsenic may gain notorieties for mainly toxicity or carcinogenicity, its cytotoxic effect on APL or EMP is profound.

Arsenic trioxide is in a class of medications called antineoplastics that has been used as a pharmaceutical agent in western and non-western medicine since first century BC. In western countries arsenicals became a therapeutic mainstay to treat syphilis before the development of penicillin. The medical relevance of it in traditional Chinese medicine (TCM) reach back 2000 years which was used to treat asthma, rheumatosis, parasitic worms, abscesses, psoriasis, and pruritus. Indian Ayurvedic herbal medicines often contain arsenic compounds and are applied in hematological malignancies.

Arsenic intoxication leads to multisystem diseases including restrictive and obstructive lung disease, polyneuropathy, renal insufficiency and renal failure, keratosis, Bowen's disease, pancreatitis, pancytopenia, diabetes mellitus, and hepatomegaly. Cardiovascular system is one of the major targets for side effects of this chemotherapeutic drug. Involvement of almost any cardiac disorder has been reported. Previously we have seen ATO induced: cardiac fibrosis, long QT syndrome, cardiotoxicity, growth inhibition and death in pulmonary artery smooth muscle cells, torsades de pointes, ventricular tachycardia, cardiac fibroblast apoptosis, complete atrioventricular block, and sudden death [13-21]. Apparently rare but now it should be considered as a possible side effect and should necessarily be part of the differential diagnosis of chemotherapeutics induced atrial fibrillation.

Amiodarone is a potent antiarrhythmic drug that is highly effective for emergency treatment of ventricular arrhythmia, life threatening arrhythmia, and atrial fibrillation. Based on various practice guidelines, amiodarone is recommended for conversion of AFib to sinus rhythm. We reviewed the existing literature to better apprehend the amiodarone for prevention of AFib in cancers. Researchers have discovered AFib to occur with an accelerated rate in patients suffering from cancers, notably in those undergoing radio-chemotherapy or tumor removal surgery. D. Farmakis et al. estimated an epidemiological evidence of AFib coincided with cancers (e.g., colorectal, esophageal, thyroid, breast) [22]. Recently, A. Asnani et al. has listed targeted tyrosine kinese inhibitors (e.g., afatinib, ceritinib, ibrutinib, ruxolitinib) therapies and plasma levels of antiarrhythmatics including amiodarone [23]. The potency of amiodarone in onco-cardiology, L. P. Riber et al. discovered that it prevents AFib in postoperative lung cancer resection in a study of 242 patients [24]. The management of arrhythmias in cancer patients whether chemotherapeutics induced, metastasis produced, or may be post-surgical complications, is difficult particularly when making clinical acumens. "Arsenic trioxide induced atrial fibrillation" is relatively rare clinical representation in extramedullary plasmacytoma. In our presenting case depicts the incidence of AFib can be possible by ATO. Initially discontinuation of ATO and oral administration of amiodarone (twice a day) has capability to improve arrhythmia significantly in a short period of time. Unfortunately, AFib recurred in our described case. In order to overcome AFib, we increased the dosage of amiodarone. Currently, we started an ATO combined with amiodarone treatment plan. No AFib relapse has been reported so far.

\section{Conclusion}

Primary lumbar disc plasmacytoma and cardiac adverse effects exacerbated by chemotherapeutics can be prevented by combined amiodarone-arsenic trioxide therapy.

\section{Consent}

Written consent was collected from our patient for adding cancer related body images, diagnostic images in the case report and publication purposes.

\section{Conflicts of interest}

It should be declared that the authors of this case report carry no conflicts of interest.

\section{References}

1. Alexiou C, Kau RJ, Dietzfelbinger H, Kremer M, Spiess JC, et al. (1999) Extramedullary plasmacytoma: tumor occurrence and therapeutic concepts. Cancer 85: 2305-14.

2. Nooka AK, Kastritis E, Dimopoulos MA, Lonial S (2015) Treatment options for relapsed and refractory multiple myeloma. Blood 125: 3085-99.

3. Murgo AJ (2001) Clinical Trials of Arsenic Trioxide in Hematologic and Solid Tumors: Overview of the National Cancer Institute Cooperative Research and Development Studies. Oncologist 6: 22-8.

4. Park WH, Seol JG, Kim ES, Hyun JM, Jung CW, et al. (2000) Arsenic Trioxide-mediated Growth Inhibition in MC/CAR Myeloma Cells via Cell Cycle Arrest in Association with Induction of Cyclin-dependent Kinase Inhibitor, p21, and Apoptosis. Cancer Res 60: 3065-71.

5. Tang B, Bajenova O, Feinman-Siegel R, Childs B, Pearse R, et al. (1998) Arsenic compounds induce apoptosis in multiple myeloma (MM), activate pro-caspase-3 but do not affect BCL2 family members. Blood 92: 638.

6. Zhang L, Liu L, Zhan S, Chen L, Wang Y, et al. (2018) Arsenic Trioxide Suppressed Migration and Angiogenesis by Targeting FOXO3a in Gastric Cancer Cells. Int J Mol Sci 19: 10.3390/ijms19123739.

7. Wang ZG, Rivi R, Delva L, König A, Scheinberg DA, et al. (1998) Arsenic trioxide and melarsoprol induce programmed cell death in myeloid leukemia cell lines and function in a PML and PML-RARa independent manner. Blood 92: 1497-504. 
8. Li YM, Broome JD (1999) Arsenic targets tubulins to induce apoptosis in myeloid leukemia cells. Cancer Res 59: 776-80.

9. Dai J, Weinberg RS, Waxman S, Jing Y (1999) Malignant cells can be sensitized to undergo growth inhibition and apoptosis by arsenic trioxide through modulation of the glutathione redox system. Blood 93: 268-77.

10. Jackson SD, Wiering BA, Herrmann AA, Hinz MA, Hanson LR (2019) Solitary bone plasmacytoma compression injury disguised as back pain: a case report. Spinal Cord Series and Cases 5: 16.

11. Pashayan R, Cavanaugh WM, Warshel CD, and Payne DR (2017) Plasmacytoma of the Cervical Spine: A Case Study. J Chiropr Med 16: 170-4.

12. Mirzashahi B, Mazoochy H, Jamnani RK, and Farzan A (2014) Contribution of Surgery in Solitary Plasmacytoma of Spine; A Case Report. Arch Bone Jt Surg 2: 121-5.

13. Zhang Y, Wu X, Li Y, Zhang H, Li Z, et al. (2016) Endothelial to mesenchymal transition contributes to arsenic-trioxide-induced cardiac fibrosis. Sci Rep 6: 33787.

14. Jiang Y, Du W, Chu Q, Qin Y, Tuguzbaeva G, et al. (2018) Downregulation of Long Non-Coding RNA Kcnq1ot1: An Important Mechanism of Arsenic TrioxideInduced Long QT Syndrome. Cell Physiol Biochem 45: 192-202.

15. Vineetha VP, Raghu KG (2019) An Overview on Arsenic Trioxide-Induced Cardiotoxicity, Cardiovasc Toxicol 19: 105-19.

16. Park WH, Han BR, Park HK, Kim SZ (2018) Arsenic trioxide induces growth inhibition and death in human pulmonary artery smooth muscle cells accompanied by mitochondrial O2•- increase and GSH depletion. Environ Toxicol 33: 833-40.

17. Unnikrishnan D, Dutcher JP, Varshneya N, Lucariello R, Api M, et al. (2001) Torsades de pointes in 3 patients with leukemia treated with arsenic trioxide. Blood 97: 1514-6.

18. Ducas RA, Seftel MD, Ducas J, Seifer C (2011) Monomorphic ventricular tachycardia caused by arsenic trioxide therapy for acute promyelocytic leukaemia. J R Coll Physicians Edinb 41: 117-8.

19. Li C, Qu X, Xu W, Qu N, Mei L, et al. (2013) Arsenic trioxide induces cardiac fibroblast apoptosis in vitro and in vivo by up-regulating TGF- $\beta 1$ expression. Toxicol Lett 219: 223-30.

20. Huang CH, Chen WJ, Wu CC, Chen YC, Lee YT (1999) Complete atrioventricular block after arsenic trioxide treatment in an acute promyelocytic leukemic patient. Pacing Clin Electrophysiol 22: 965-7.

21. Westervelt P, Brown RA, Adkins DR, Khoury H, Curtin P, et al. (2001) Sudden death among patients with acute promyelocytic leukemia treated with arsenic trioxide. Blood 98: 266-71.

22. Farmakis D, Parissis J, Filippatos G (2014) Insights Into Onco-Cardiology: Atrial Fibrillation in Cancer. J Am Coll Cardiol 63: 945-53.

23. Asnani A, Manning A, Mansour M, Ruskin J, Hochberg EP, et al. (2017) Management of atrial fibrillation in patients taking targeted cancer therapies. Cardio-Oncology 3: 2.

24. Riber LP, Christensen TD, Jensen HK, Hoejsgaard A, Pilegaard HK (2012) Amiodarone Significantly Decreases Atrial Fibrillation in Patients Undergoing Surgery for Lung Cancer. Ann Thorac Surg 94: 339-46.

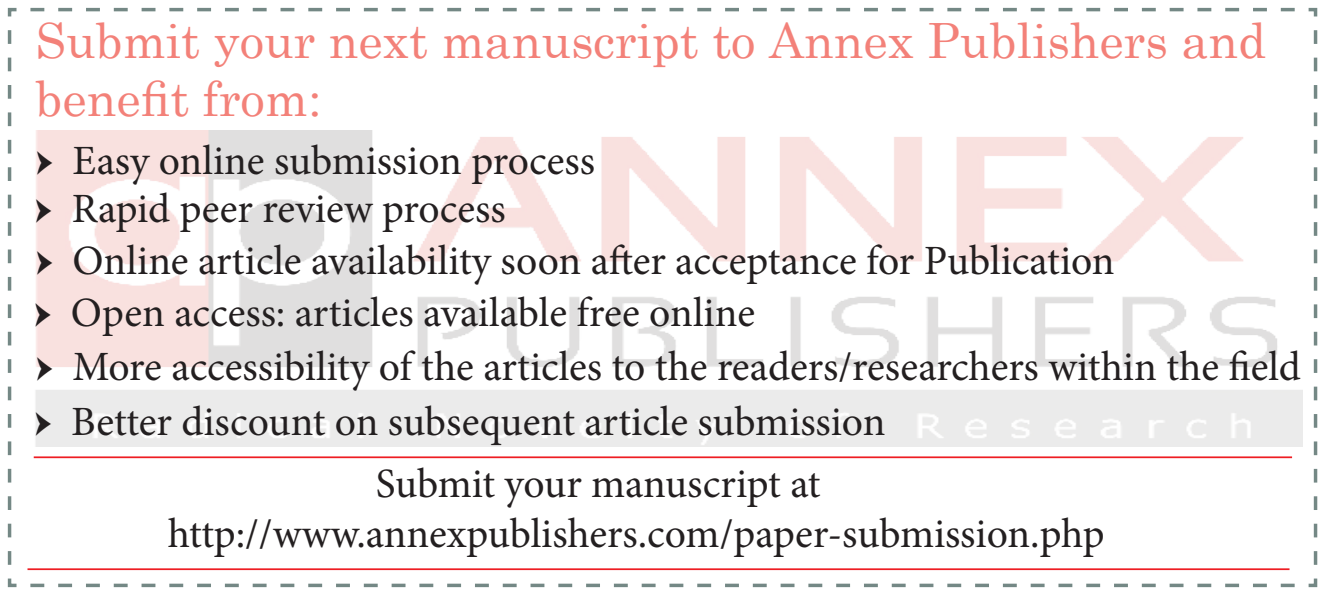

